ARTICLE

https://doi.org/10.1038/s41467-019-12134-4

\title{
Single- and multi-component chiral supraparticles as modular enantioselective catalysts
}

\author{
Si Li, ${ }^{1,2,3}$, Juan Liu ${ }^{4}$, Naomi S. Ramesar ${ }^{2,3}$, Hendrik Heinz ${ }^{4}$, Liguang $\mathrm{Xu}^{1}$, Chuanlai Xu (D) ${ }^{1}$ \&
}

Nicholas A. Kotov (10) 2,3,5,6

Nanoscale biological assemblies exemplified by exosomes, endosomes and capsids, play crucial roles in all living systems. Supraparticles (SP) from inorganic nanoparticles (NPs) replicate structural characteristics of these bioassemblies, but it is unknown whether they can mimic their biochemical functions. Here, we show that chiral ZnS NPs self-assemble into 70-100 nm SPs that display sub-nanoscale porosity associated with interstitial spaces between constituent NPs. Similarly to photosynthetic bacterial organelles, these SPs can serve as photocatalysts, enantioselectively converting $L$ - or $D$-tyrosine (Tyr) into dityrosine (diTyr). Experimental data and molecular dynamic simulations indicate that the chiral bias of the photocatalytic reaction is associated with the chiral environment of interstitial spaces and preferential partitioning of enantiomers into SPs, which can be further enhanced by coassembling ZnS with Au NPs. Besides replicating a specific function of biological nanoassemblies, these findings establish a path to enantioselective oxidative coupling of phenols for biomedical and other needs.

\footnotetext{
${ }^{1}$ State Key Lab of Food Science and Technology, International Joint Research Laboratory for Biointerface and Biodetection, School of Food Science and Technology, Jiangnan University, Wuxi 214122 Jiangsu, People's Republic of China. ${ }^{2}$ Department of Chemical Engineering, University of Michigan, Ann Arbor, MI 48109, USA. ${ }^{3}$ Biointerfaces Institute, University of Michigan, Ann Arbor, MI 48109, USA. ${ }^{4}$ Department of Chemical and Biological Engineering, University of Colorado Boulder, Boulder, Colorado, USA. ${ }^{5}$ Department of Materials Science, University of Michigan, Ann Arbor, MI 48109, USA.

${ }^{6}$ Macromolecular Science and Engineering, University of Michigan, Ann Arbor, MI 48109, USA. Correspondence and requests for materials should be addressed to L.X. (email: xuliguang2006@126.com) or to N.A.K. (email: kotov@umich.edu)
} 
$\mathrm{N}$ anoscale biological assemblies are composed of a diverse spectrum of (bio)organic components, which are common in all living systems ${ }^{1,2}$. They have characteristic dimensions ranging from 50 to $500 \mathrm{~nm}$ and are exemplified by viral capsids ${ }^{3}$, endosomes ${ }^{4}$, exosomes, carboxysomes ${ }^{5}$, azurophilic granules $^{6}$, light-harvesting bacterial organelles ${ }^{7}$, cellular vesicles ${ }^{8}$, intraluminal vesicles, stress granules ${ }^{9}$, and a wide spectrum of intracellular membraneless compartments. These self-contained biological spheroids, chemically related to micelles, are responsible for numerous intracellular and extracellular functions, including high-efficiency site-specific transport ${ }^{2,8}$, cargo protection $^{3}$, signaling ${ }^{10}$, catalysis ${ }^{11}$, light absorption, biomolecular filters, autophagy ${ }^{9}$, and protein folding ${ }^{12,13}$. Nanoassemblies with chemically similar functions are needed for different technological areas, exemplified by drug delivery, medicine, biotechnology, solar fuels, and $\mathrm{CO}_{2}$ reduction. Bioinspired nanoscale assemblies can be produced from human-made components, including recombinant proteins and amphiphilic biomacromolecules ${ }^{14,15}$. Similar spheroidal structures can also be made using DNA nanotechnology ${ }^{16}$. However, the ex-vivo use of all these nanoassemblies requires the negotiation of both the benefits and problems related to biomolecular building blocks. One of the problems is their stability in reactive media that diminishes the longevity of proteins ${ }^{17}, \mathrm{RNA}^{18}$, and $\mathrm{DNA}^{19}$. Since inorganic nanoparticles (NPs) have essentially the same ability to spontaneously assemble into complex superstructures ${ }^{20}$, it is possible to recreate some of these assemblies and functions from inorganic components that are robust and inexpensive, which is explored in this study.

Inorganic constructs that are conceptually similar to nanoscale assemblies from (bio)organic subunits are represented by supraparticles (SPs), formed from 50-500 individual NPs ${ }^{21,22}$ Similar to micelles, they self-assemble because of the interplay between short-range attractive and long-range repulsive forces associated with the constituent building blocks ${ }^{21,23}$. Gradually increased electrostatic repulsion of SPs terminates the attachment of new NPs when a certain threshold is reached; this leads to self-limited growth and thus size uniformity. SPs display sizes and geometries reminiscent of biological nanoassemblies, and can adopt a range of morphologies from fairly simple structures, such as nanospheres $^{24}$ or nanoshells ${ }^{25}$ to fairly sophisticated left- and righthanded helices ${ }^{26}$. SPs made of NPs are structurally flexible, enabling their modular engineering from different constitutive units, which are evidenced by the inclusion of biological nanoscale components into the structures of SPs.

Among the many known functions of biological nanoassemblies, catalysis is perhaps the most common one. Chiral catalysis takes place in many of these nanoscale "organs", including chloroplasts $^{27}$, endoplasmic reticulum ${ }^{28}$, and endosomes ${ }^{4}$. Following the ever-growing demand for chiral intermediates ${ }^{29}$ and novelty of chiral NPs, it will be important to investigate their advantages compared to homogeneous chiral catalysts ${ }^{30}$. Several recent studies on NPs as heterogeneous chiral catalysts have demonstrated their synthetic simplicity and catalytic activity ${ }^{31-34}$. However, stereo-selectivity in these nanoscale constructs is provided by complex metalorganic ligands, which leads to vulnerability of these catalysts to ligand replacement, oxidation, and cross-linking. In a way, inorganic core of NPs served in these studies serves mostly as a carrier rather than as the source of chiral bias. Conversely, individual NPs with chiral ligands that are short and simple fail to display observable enantioselectively ${ }^{35}$.

SPs are especially desirable as chiral catalysts because they can be constructed from metal, ceramic, and semiconductor $\mathrm{NPs}^{36-38}$, integrating different components into one structural unit optimized for stability, enantioselectivity, and energy efficiency. Here we show that SPs constructed from chiral NPs display catalytic activity and enantioselectivity for the photoinduced oxidation of tyrosine (Tyr) known to be one of the difficult reactions for chiral catalysis. The photocatalytic processes in inorganic SPs mimic Tyr dimerization in photosynthetic bacterial organelles $^{39}$, demonstrating functional replication of biological nanoscale assemblies. Furthermore, enantioselectivity for Tyr-Tyr has not been reported due to lack of efficient catalysts $^{40,41}$ while asymmetric oxidative coupling of other phenols was shown to be difficult to achieve even with sophisticated metal-organic catalysts ${ }^{42,43}$. The catalytic functions in these assemblies can be optimized by taking advantage of SP modularity, combining NPs with different chemical and optical properties.

\section{Results}

Key problems of nanoassemblies for chiral catalysis. While numerous chiral NPs and their assemblies have been reported over the last two decades, these studies were primarily focused on understanding and maximizing chiroptical activity $33-35,44,45$. When designing NP assemblies to display chiral bias, one encounters the fundamental problem of the mismatch in scales between the chiral geometries characteristic of small molecules needed for pharmaceuticals, liquid crystals, chiral polymers, and other needs versus the chiral shapes of NPs. For example, in the classical case of tartrate, only five atoms constitute the chiral atomic group with a dimension of only a few angstroms, whereas the number of atoms in chiral NPs and constructs thereof can exceed tens of thousands, with dimensions from a few nanometers to $100 \mathrm{~nm}$. Additionally, NPs always have structural imperfections and variability in their atomic and molecular scales that inevitably lead to difficulties with the realization of fine angstrom-scale stereochemical control typically expected for chiral catalysts, albeit this may not be always needed.

We addressed these problems using SPs formed from semiconductor NPs as the basic structural modules ${ }^{25}$. The interstitial spaces between NPs in these nanospheroids are subnanometer in width and are comparable to the dimensions of small molecules serving as catalytic substrates. However, there is a tendency for NPs to form assemblies with nearly epitaxial merging of crystal lattices ${ }^{26,46,47}$. While useful in electronics ${ }^{48}$ and energy storage applications ${ }^{49}$, this is detrimental to chiral catalysis because it eliminates the SP's chirality in the nanoscale pores. Therefore, NPs with relatively high recrystallization energies and interatomic bonds with fairly strong covalent character are preferred.

Assembly of chiral SPs. Assembly of chiral SPs can be accomplished by assembling ZnS NPs with an average diameter, $d_{\text {TEM }}=3 \pm 0.7 \mathrm{~nm}$, stabilized by $\mathrm{L}^{-}$or D-penicillamine (Pen) (Fig. 1a); when needed, the rac-form of Pen was also utilized, resulting in NPs of similar size and polydispersity. The synthesized chiral SPs will be referred to as L-, D-, or rac-SPs, depending on the chirality of the surface ligand tethered to the NPs. Unlike optical centers formed by $s p^{3}$ hybridized carbon atoms, chiral NPs and their assemblies have multiple scales of mirror asymmetry ${ }^{29}$. Thus, L-, D-, or rac-notations refer only to the preparative methods rather than to geometrical attributes of the nanoassemblies.

Pen-ZnS NPs were synthesized and assembled into SPs by incubation at $95^{\circ} \mathrm{C}$ for $3-6 \mathrm{~h}$ (see Methods). The individual $\mathrm{ZnS}$ NPs exhibited irregular morphology as seen from the highresolution transmission electron microscopy (HR-TEM) images (Supplementary Fig. 1). Association of NPs is accompanied by the concomitant increase of the absolute value of electro-kinetic zetapotential $(\zeta$ ) (Supplementary Fig. 2). The point where it reaches a plateau corresponds to the formation of SPs, and for Pen-ZnS 

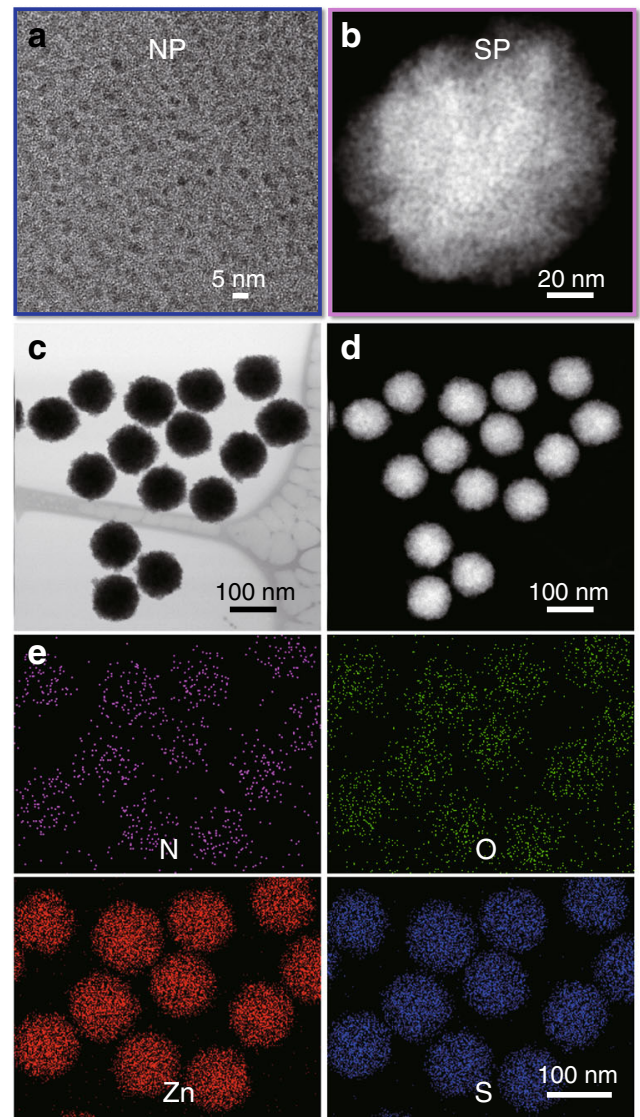

Fig. 1 Pen-stabilized chiral NPs and SPs. a TEM image of L-Pen-stabilized ZnS NPs. b Magnified HAADF-STEM image of L-ZnS SPs that assembled from the chiral NPs shown in a. c HAADF-STEM image and $\mathbf{d}$ Bright-fieldSTEM image of L-ZnS SPs $(100 \pm 4 \mathrm{~nm})$ with large scale. e Elemental mapping images of nitrogen, oxygen, zinc, and sulfur ZnS SPs

NPs, this occurs in $3 \mathrm{~h}$, when $\zeta$ reaches $-47 \mathrm{mV}$. The resulting SPs displayed a spherical morphology with diameters determined by TEM and dynamic light scattering (DLS) of $d_{\mathrm{TEM}}=100 \pm$ $3 \mathrm{~nm}$ and $d_{\mathrm{DLS}}=105 \pm 10 \mathrm{~nm}$, respectively (Fig. $1 \mathrm{~b}-\mathrm{d}$, Supplementary Figs. 3 and 4). They also showed high monodispersity with standard deviation $<6 \%$, which indicates the quasiequilibrium state of the SPs. Elemental mapping confirmed the composition of SPs, indicating the presence of $\mathrm{ZnS}$ and Pen (Fig. 1e). The presence of Pen ligands in ZnS SPs was also confirmed by the Fourier-transform infrared spectroscopy (FTIR) and Raman spectra (Supplementary Figs. 5 and 6). The typical peaks at $3000-3300 \mathrm{~cm}^{-1}$ are attributed to the stretching vibration of $\mathrm{N}-\mathrm{H}$ of Pen attached to the surface of $\mathrm{ZnS}$ NPs. The peaks at $1450-1700 \mathrm{~cm}^{-1}$ and $2200-2450 \mathrm{~cm}^{-1}$ are attributed to $\mathrm{C}-\mathrm{H}$ bending and $\mathrm{S}-\mathrm{H}$ stretching vibrations of Pen. The peaks associated with S-H bonds could not be observed for Pen$\mathrm{ZnS}$ SPs as expected, due to the formation of S- $\mathrm{ZnS}$ bonds.

Individual NPs could be identified in the assembled SPs by high-angle annular dark-field scanning TEM (HAADF-STEM) images (Fig. 1b, Supplementary Fig. 9) and high-resolution STEM images (Supplementary Figs. 7and 8). The pore size of the SPs are below $2 \mathrm{~nm}$ (Supplementary Fig. 10b), which are comparable with the sizes of reactive centers on many enzymes ${ }^{50}$. The X-ray diffraction (XRD) spectra for SPs revealed [0036], [110], [1136], [1211] peaks of ZnS in SPs (Supplementary Fig. 11).

Chirality of assembled SPs. ZnS NPs with L- and D-Pen as surface ligands displayed circular dichroism (CD) peaks at 215 and
$240 \mathrm{~nm}$ (Fig. 2a, b). Compared with the CD spectra of Pen alone (Supplementary Fig. 12), a new CD peak appeared from 240 to $300 \mathrm{~nm}$; this may be attributed primarily to electronic hybridization between the molecular orbitals of NPs and those of chiral ligands. The local distortions of the $\mathrm{ZnS}$ surface due to attachment of Pen ligands may contribute to the CD spectra, too.

Assemblies of individual NPs into ordered structures may exhibit CD signatures of the chiral geometries of NPs that may or may not possess a distinctively chiral shape, such as a helix. Unlike the individual NPs, the chiroptical activity of the prepared SPs included an additional band in the $280-340 \mathrm{~nm}$ region. Note that the energies of electronic state developing as a result of hybridization of chiral states from amino acid residues attached to the NP surface are dependent on the environment. They are different for the individual NPs and that in the interstitial spaces and thus are located at longer wavelengths. One can also expect that CD peak for such electronic states will be broad as they are, indeed, in Fig. 2d.

Assembly of multi-component chiral SPs. Similar to other terminal assemblies, SPs can be made from several different NPs so that they may offer a combination of different functionalities. For instance, the co-assembly of NPs from metals and semiconductors is a simple process that results in the combination of NPs with plasmonic and excitonic properties, which should facilitate photocatalytic processes. Gold NPs carrying glutathione (GSH) units as surface ligands were chosen as complementary unimers to the Pen-stabilized ZnS NPs for the assembly of plasmon-exciton SPs because of their similarity in size. When $\mathrm{GSH}-\mathrm{Au} \mathrm{NPs}$ with diameters of $2 \pm 0.3 \mathrm{~nm}$ (Supplementary Fig. 13) were added to a dispersion of $\mathrm{ZnS}$ SPs and incubated for $3 \mathrm{~h}$ at $95^{\circ} \mathrm{C}$, hybrid $\mathrm{ZnS}-\mathrm{Au}$ SPs containing both types of constituent blocks were obtained (Fig. 3a). The $\zeta$-potential of the multi-component SPs stabilized at a high value of $\sim-44 \mathrm{mV}$ after $4 \mathrm{~h}$ of incubation, indicating the successful formation of SPs (Supplementary Fig. 14). Note that the $\zeta$-potential characteristic of the completed self-assembly process is nearly identical to that found for ZnS nanospheroids (Supplementary Fig. 2). The average diameter of $\mathrm{ZnS}-\mathrm{Au}$ SPs was $d_{\mathrm{TEM}}=75 \pm 3 \mathrm{~nm}$ (Fig. 3b; Supplementary Fig. 15), which was about $25 \%$ smaller than the diameter of single-component nanoassemblies. Elemental mapping confirmed the presence of gold NPs in these SPs (Fig. 3c). $\mathrm{ZnS}-\mathrm{Au}$ SPs displayed CD signals from 200 to $550 \mathrm{~nm}$ (Fig. 3d). The polarity of chiroptical activity at $220 \mathrm{~nm}$ for L-ZnS-Au, that is, those where ZnS NPs were made with L-Pen, was opposite compared with the CD spectra of single-component $\mathrm{ZnS}$ SPs (Fig. 2d) and GSH-Au NPs (Supplementary Fig. 13b). Thepolarity reversal may have multiple physical origins ${ }^{29}$ and likely represents a convolution of various effects. It may be attributed to both strong chemical (such as hydrogen bonding between the surface ligands) and physical (such as plasmon-exciton hybridization) interactions between $\mathrm{ZnS}$ and $\mathrm{Au}$ NPs in the nanoassemblies.

SPs as modular photocatalysts. The chirality of SPs can potentially endow them with enantioselectivity in catalysis. Realization of enantioselective photocatalysts would be particularly promising because the photoinduced reactions require both optical activity and robustness of inorganic NPs to prevent their photodecomposition. The conversion of the amino acid Tyr, which can be monitored using its strong photoluminescence (PL) peak at $306 \mathrm{~nm}$, was used as a chiral catalytic substrate. Furthermore, Tyr can be photo-oxidized to dityrosine $(\text { diTyr })^{51-55}$ or dihydroxy phenylalanine (DOPA) ${ }^{53}$, with characteristic PL peaks at 414 and $320 \mathrm{~nm}$ (Supplementary Fig. 16), respectively. The realization of 

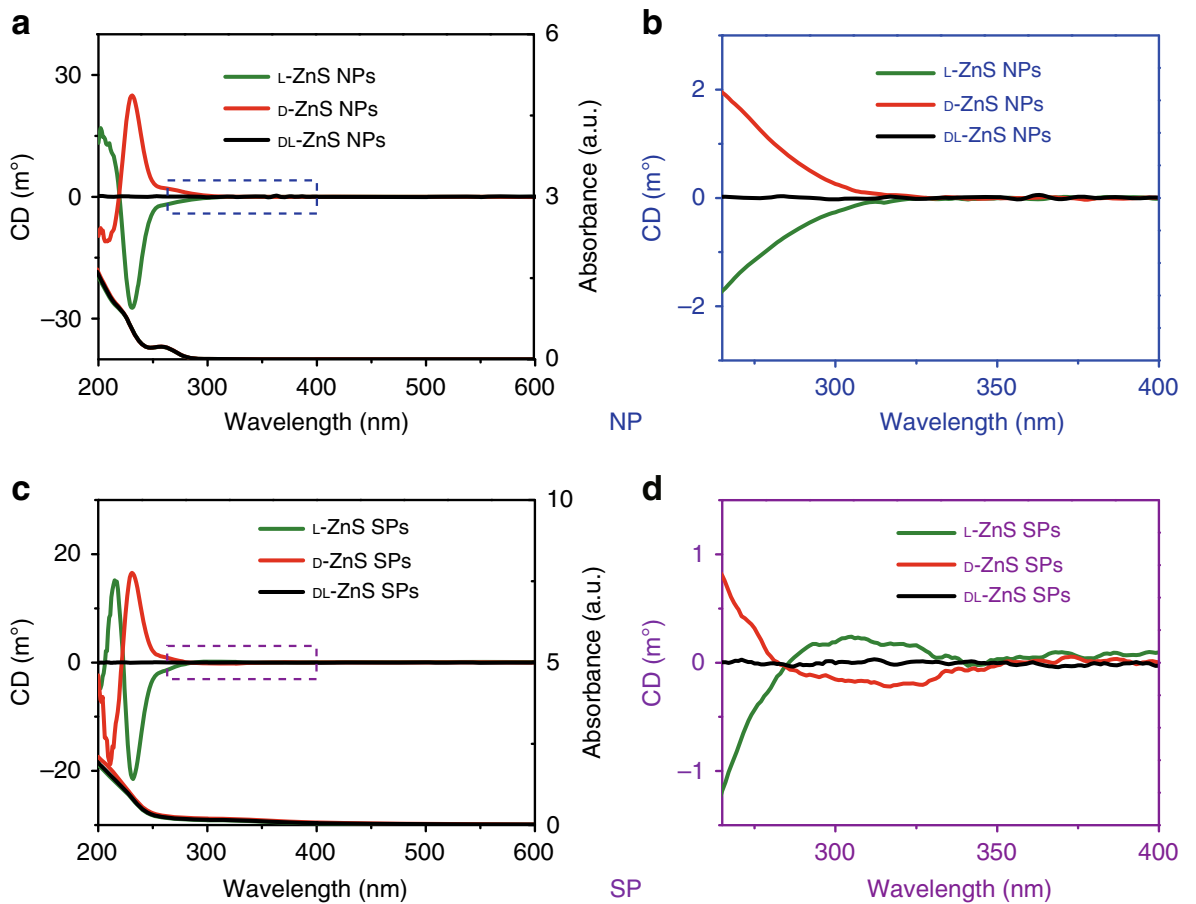

Fig. 2 CD spectra of Pen-stabilized ZnS NPs and ZnS SPs. a CD spectra and UV spectra of ZnS NPs with Pen surface ligands of different handedness. b Enlarged CD spectra of ZnS NPs from 265 to $400 \mathrm{~nm}$. c CD spectra and UV spectra of ZnS SPs with Pen surface ligands of different handedness. d Enlarged CD spectra of ZnS SPs from 265 to $400 \mathrm{~nm}$

asymmetric photo-oxidative coupling of Tyr to diTyr would be particularly impactful from the standpoints of both chiral catalysis and pharmaceutical intermediates because enantioselectivity for such reactions was so far challenging to realize ${ }^{40-42}$.

The photocatalytic reactions in all experimental series were initiated by illumination at $\mathrm{pH} 8.0$ with UV light at $315-400 \mathrm{~nm}$, which was efficiently absorbed by $\mathrm{ZnS}$ and $\mathrm{ZnS}-\mathrm{Au}$ SPs (Figs. 2c and 3d). Illumination of Tyr without SPs yielded a nearly negligible change in PL spectra (Fig. 4a, b). When ZnS SPs were added to the Tyr solution, a strong increase in PL intensity was observed at $414 \mathrm{~nm}$, which is characteristic of the accumulation of diTyr (Fig. 4c-f $)^{52,54}$. DiTyr formation was accompanied by a decrease of PL intensity at $306 \mathrm{~nm}$ as expected considering the consumption of Tyr. While diTyr was the main product of the photocatalytic conversion of Tyr, the formation of DOPA may also be expected, which explained the red-shift of the emission peak at $306 \mathrm{~nm}$ in Fig. 4 as the photolysis proceeded.

Analysis of temporal profiles of diTyr emission, $I_{414}(t)$, allowed us to evaluate the chiral preferences of the SP catalysts. ZnS SPs carrying L-Pen preferentially catalyzed L-Tyr, while SPs made from D-Pen preferentially catalyzed D-Tyr (Fig. 4g). The degree of enantioselectivity can be associated with preferential partitioning of enantiomers of Tyr into the SPs, which was confirmed by an enantioselective adsorption of Tyr enantiomers after exposure of rac-Tyr solution to L- or D-ZnS SPs. The effect of preferential adsorption of enantiomers on chiral substrates and phases was observed for some other systems ${ }^{44,56-58}$, but, to the best of our knowledge, not for SPs or any other nanoscale assemblies of NPs. Calibration of the $\mathrm{CD}$ spectra with respect to the total concentrations of each enantiomer (Supplementary Figs. 17 and 18) yields preferential partitioning of L-Tyr and D-Tyr into L- and $\mathrm{D}$-ZnS-SPs as $38.4 \pm 1.8 \%$ and $36.3 \pm 2.4 \%$ over the other isomer, respectively, while that of $\mathrm{L}^{-}$and $\mathrm{D}-\mathrm{ZnS}$ NPs was $11.5 \% \pm 3.1 \%$ and $15.1 \% \pm 3.4 \%$ over the other isomer. Thus, the enantioselective recognition was vividly enhanced via assembling chiral NPs into SPs. The chiral preferences of SP-substrate interactions match those observed in photocatalysis, which is consistent with the enantioselective physisorption of substrates with NPs and SPs (Fig. 4g). The individual ZnS NPs with L- or D-ligands did not show obvious catalytic preference for enantiomeric Tyr, and the catalytic activity is also lower than that of the SPs (Supplementary Fig. 19). Both the ZnS NPs and ZnS SPs retain excellent stability in the course of photocatalysis (Supplementary Figs. 20 and 21). Importantly, the photochemical stability of chiral ligands on SPs is markedly better than that on NPs. In fact, no decomposition of Pen was observed in SPs after intense illumination for $36 \mathrm{~h}$ (Fig. 4h).

When Tyr was illuminated in the presence of $\mathrm{ZnS}-\mathrm{Au}$ SPs, the fluorescence intensity of the $306 \mathrm{~nm}$ band decreased dramatically during the illumination (Fig. 5a, b), which indicated a large enhancement of the efficiency of photocatalytic conversion of Tyr after incorporation of Au NPs. Note, however, that the rise of the $414 \mathrm{~nm}$ peak associated with diTyr was smaller in $\mathrm{ZnS}-\mathrm{Au}$ than in ZnS SPs (Figs. 4c-f and $5 \mathrm{a}, \mathrm{b}$ ), pointing to the simultaneous enhancement of diTyr conversion into non-luminescent products.

As a result of the higher catalytic activity of ZnS-Au SPs, weaker PL signature of diTyr, and greater complexity of the products than in single-component SPs, the chemical composition of products of photocatalysis were investigated using additional analytical techniques. High-performance liquid chromatography-mass spectrometry (HPLC-MS) confirmed the efficiency of the photoinduced oxidative coupling of Tyr by ZnS-Au SPs. The peaks in extracted ion chromatography (EIC) provided spectra tracings that concomitantly verified the conversion of Tyr to diTyr and DOPA (Fig. 5c). The gradual stabilization of the EIC peaks corresponding to diTyr and DOPA for the reaction time from 18 to $32 \mathrm{~h}$ confirmed the ability of multi-component SPs to subsequently oxidize diTyr and DOPA, which was not the case for single-component SPs.

Similarly to $\mathrm{ZnS}$ SPs, the temporal dependence of PL intensity at $306 \mathrm{~nm}$ revealed that L-ZnS-Au SPs preferentially catalyzed the photo-oxidation of L-Tyr over D-Tyr (Fig. 5d). The 

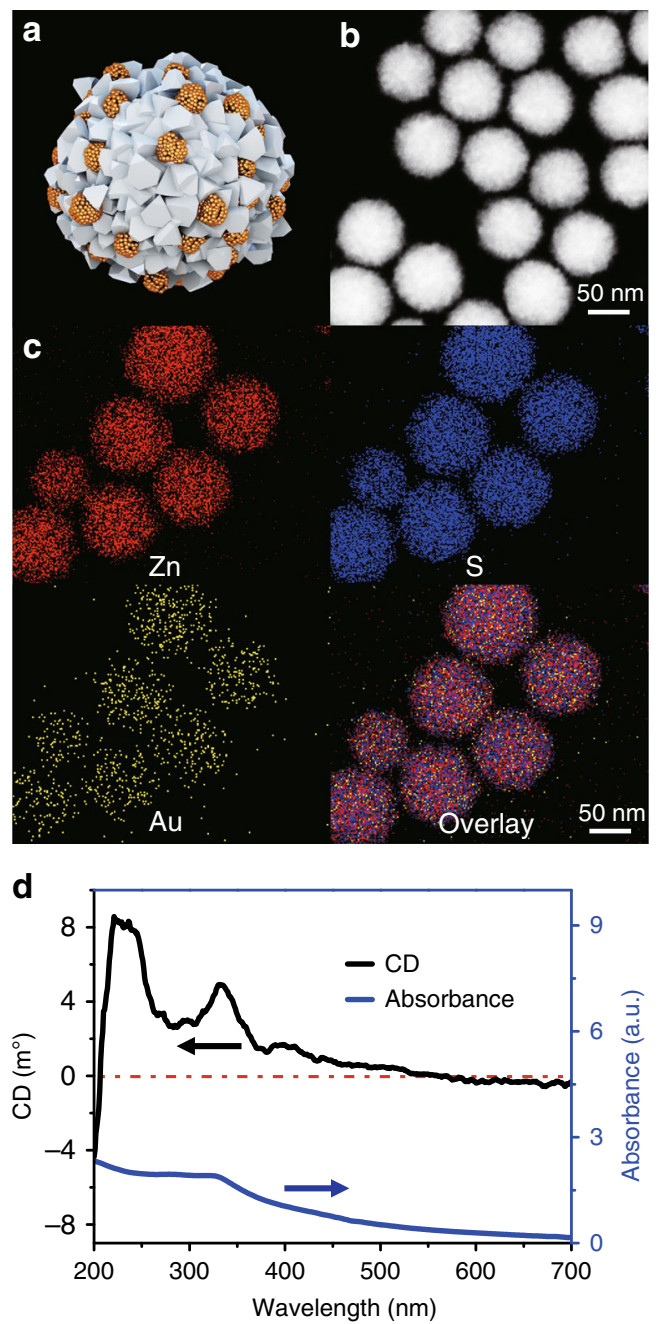

Fig. 3 Morphology and spectra characterization of chiral ZnS-Au SPs. a Model of multi-component L-ZnS-Au SPs. b HAADF-STEM images of multi-component L-ZnS-Au SPs. c Elemental mapping of L-ZnS-Au SPs of zinc, gold, and sulfur, respectively. $\mathbf{d} C D$ and UV spectra of multicomponent L-ZnS-Au SPs

association of enantioselectivity of the photocatalysis with preferential partitioning of a specific enantiomer was confirmed for the multi-component SPs. When incubated with rac-Tyr, L$\mathrm{ZnS}-\mathrm{Au}$ SPs prefer to adsorb L-Tyr in the amount $44.2 \pm 1.7 \%$ greater than that for D-Tyr (Supplementary Figs. 18 and 22). Selective adsorption of Tyr enantiomers in $\mathrm{ZnS}$ and $\mathrm{ZnS}-\mathrm{Au}$ SPs indicates that ZnS NPs with Pen surface ligands are primarily responsible for the chiral bias of the photo-oxidation process. The improved catalytic performance of $\mathrm{Au}-\mathrm{ZnS}$ SPs can be attributed to more facile separation of photogenerated electrons and holes and the reduced band gap of ZnS. The photogenerated electrons prefer $\mathrm{Au}$ surface rather than $\mathrm{ZnS}$. The holes, however, will mainly stay on the $\mathrm{ZnS}$ nanoparticles, which will reduce the recombination of the electron-hole pairs and enhance the catalytic efficiency.

The photochemical stability of chiral ligands on $\mathrm{Au}-\mathrm{ZnS}$ SPs was shown to exceed those in the dispersion of individual $\mathrm{ZnS}$ and $\mathrm{Au}$ NPs (Fig. 5e). No degradation of Pen and GSH was observed for illumination for $60 \mathrm{~h}$ whereas marked reduction of CD intensity of the peaks corresponding to both peptides was observed for free NPs over the same time.

There are several chemical mechanisms why the stability of the ligands would increase in SPs compared to chiral photocatalysts based on single NPs or traditional organometallic compounds. First, SPs afford utilization of short and robust chiral ligands, such as individual amino acids as molecular structures responsible for enantioselectivity, as opposed to complex and sensitive high molecular weight constructs. The higher the molecular weight of the surface ligands, the greater number of conformational degrees of freedom, and the greater the possibility of chemical damage due to photonically or thermally initiated reactions. Second, the light in SP is absorbed by a subset of NPs. These can be the NPs in the surface or Au NPs in ZnS or Zn-S-Au SPs, respectively. Unlike single NPs or the traditional chiral photocatalysts, the excitation can be efficiently transferred between several NPs due to their closeness in the SP assemblies. The resonance coupling of the excited states in SPs is advantageous for the stability of the chiral photocatalysts because on chiral ligands on the internal NPs are better protected against damage than those on the SP surface. Third, SPs represent a self-assembled hybrid composite where $\mathrm{ZnS}$ cores and organic ligands on their surface constitute the mineral and organic components, respectively. Multiple experimental data indicate that the organic molecules in the composite display increased resilience to oxidation, thermal decomposition, or hydrolysis ${ }^{59-61}$. This property represents the fundamental structural advantage of SPs as chiral photocatalysts.

Molecular dynamics simulation. Molecular dynamics simulation can be a powerful tool for investigation of the atomic scale dynamics and molecular recognition properties at NP surfaces, and was applied here to obtain insight into the recognition of ( $\mathrm{L}$ or D)-Tyr in aqueous solution on (L or D)-Pen-ZnS NPs (Fig. 6). The enantioselectivity of Pen-ZnS surfaces were evaluated using the binding frequency of Tyr enantiomers to the Pen ligands on single NPs and model nanoassemblies from four NPs (Supplementary Figure 23) during $50 \mathrm{~ns}$ and 20 ns, respectively, molecular dynamics simulations in explicit water at $\mathrm{pH} 7.0$ (Fig. 6). Binding for both single- and four-NP systems decreases in the order D-Pen/D-Tyr $>$ L-Pen/L-Tyr $>$ L-Pen/D-Tyr $>$ D-Pen-L-Tyr, which matches with the reaction rate for Tyr consumption and diTyr formation (Fig. $4 \mathrm{~g}$ from measurements). The most preferable binding interactions occur between $-\mathrm{COO}^{-}$ions in Pen and $-\mathrm{NH}_{3}{ }^{+}$groups in Tyr, as well as between $-\mathrm{NH}_{3}{ }^{+}$groups in Pen and $-\mathrm{COO}^{-}$groups in Tyr in all four systems (Fig. 6b). The binding involves ion pairing and hydrogen bonds. Once two binding contacts simultaneously form between a Pen ligand and Tyr, Tyr will remain bound in excess of $10 \mathrm{~ns}$. When only one of the binding contacts forms, the binding status is more dynamic and lasts only 1-2 ns. As the aromatic side group of Tyr approaches the bare $\mathrm{ZnS} \mathrm{NP}$ surface, the $-\mathrm{OH}$ group may temporarily bind to the $\mathrm{ZnS}$ surface and disengage binding to Pen via ion pairing. Differences in binding properties for different chirality combinations of ligands and Tyr molecules are related to distinct relative orientations of the carboxylate and ammonium groups of Pen on the particle surface (inward/outward depending on $\mathrm{D} / \mathrm{L}$ ), as well as specific interactions between neighbor ligands. Tyr binds selectively to these chiral ligand surfaces and experiences some geometry differences in match depending on (D) or (L) configuration. In addition, the binding competition between the charged groups in $\mathrm{Tyr}\left(-\mathrm{NH}_{3}{ }^{+}\right.$and $\left.-\mathrm{COO}^{-}\right)$with Pen ligands and the aromatic side groups in Tyr with uncovered areas of the ZnS NP surface demonstrates that the $\mathrm{pH}$ value and the density of surface ligands can affect the binding affinity. Diffusion rate of L-Tyr in the center of four-NP assembly was found to be $0.22 \pm 0.05$ and $0.34 \pm 0.12 \mathrm{~A} / \mathrm{ns}$ for $\mathrm{D}$ - and L-Pen-ZnS models, respectively. Concurrently, the diffusion rates of $\mathrm{L}-\mathrm{Tyr}$ on the 

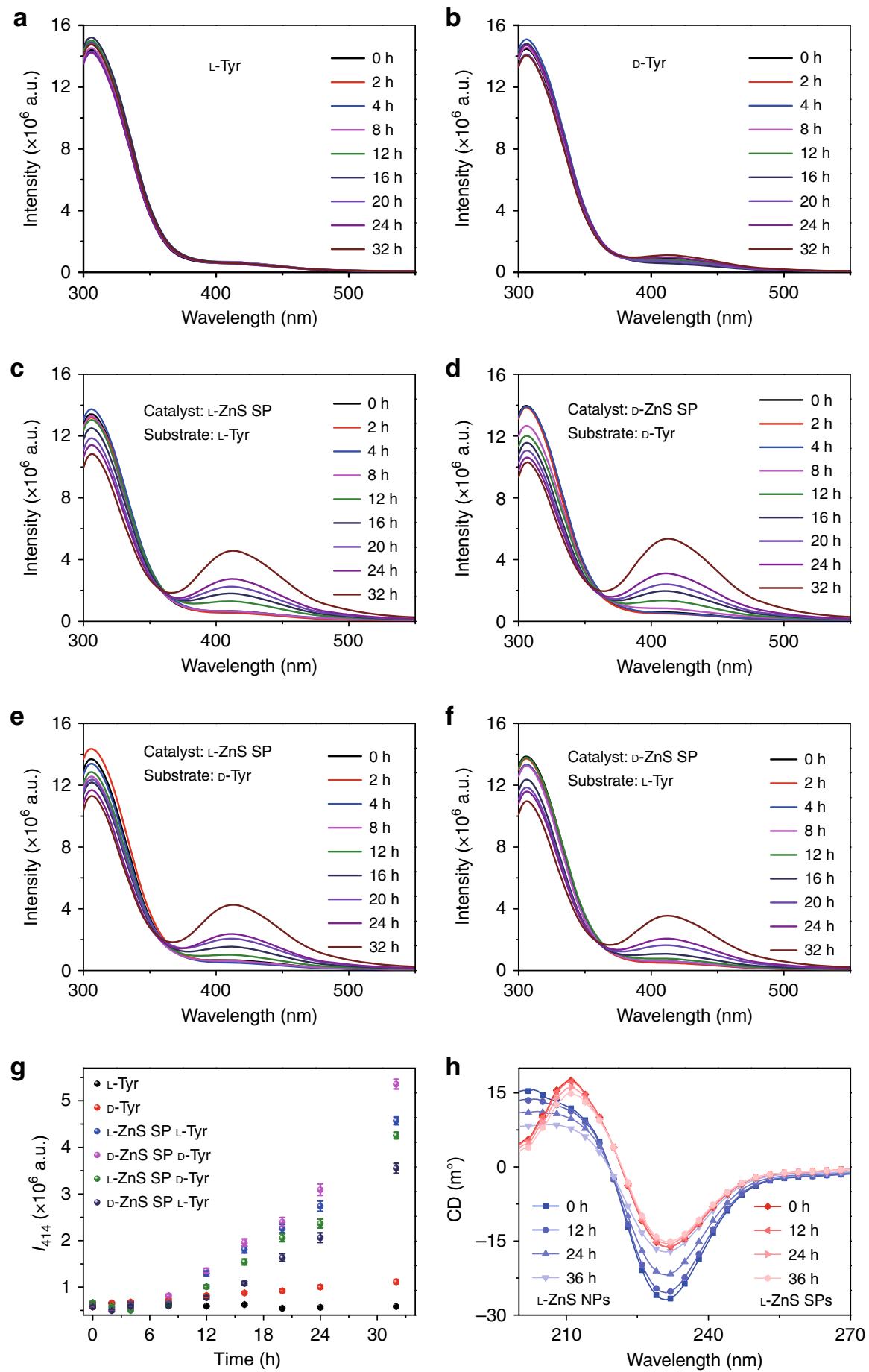

Fig. 4 Catalisis and stability of ZnS SPs for photoinduced oxidative coupling of L-Tyr and D-Tyr. Fluorescence spectra of L-Tyr or D-Tyr after $\mathbf{a} L-T y r$, $\mathbf{b} D-T y r$, c L-Tyr with L-ZnS SPs, and $\mathbf{d} D$-Tyr with D-ZnS SPs. e L-Tyr with D-ZnS SPs and $\mathbf{f} D$-Tyr with L-ZnS SPs being illuminated with different periods of time in the absence or presence of ZnS SPs. $g$ The dependence of PL intensity at $414 \mathrm{~nm}$ on the time of photocatalytic reaction with ZnS SPs of different handedness of the catalyst and the substrate. $\mathbf{h}$ CD spectra of the mixtures of ZnS NPs and ZnS SPs after UV illumination $(115 \mathrm{~V}, 50 / 60 \mathrm{~Hz}, 0.16 \mathrm{~A})$ for different times

sides of the nanoassembly were much faster $-2.2 \pm 0.6$ and and 1.8 $\pm 0.5 \mathrm{~A} / \mathrm{ns}$ for the same models. This observation supports the attribution of experimentally observed enhancement of both catalytic activity and enantioselectivity in SPs vs. NPs to extended residence time of substrate inside the catalytic particle.

\section{Discussion}

Single- and multi-component SPs with size and uniformity comparable to biological nanoassemblies were assembled from chiral semiconductor and metallic NPs mimicking both structure and functions of their biological analogs, for instance photosynthetic organelles of bacteria and, to a lesser extent, redox reactions in azurophilic granules. SPs showed efficient photocatalysis of Tyr with enantioselectivity determined by the chiral preferences of L- and D-Tyr to interact with individual NPs and penetrate into the interstitial spaces between them. It needs to be pointed out that enantioselectivity of oxidative phenol coupling, which is Tyr-Tyr dimerization is representative of, is both 
a

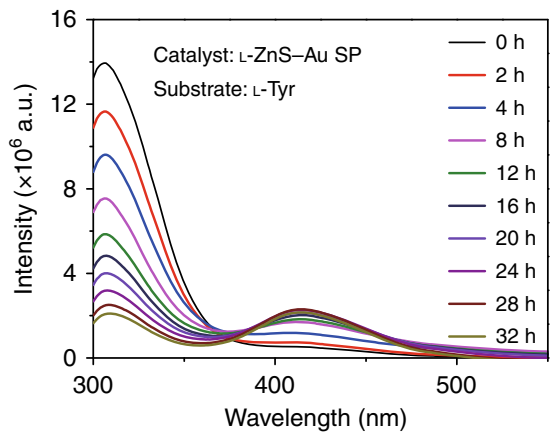

b

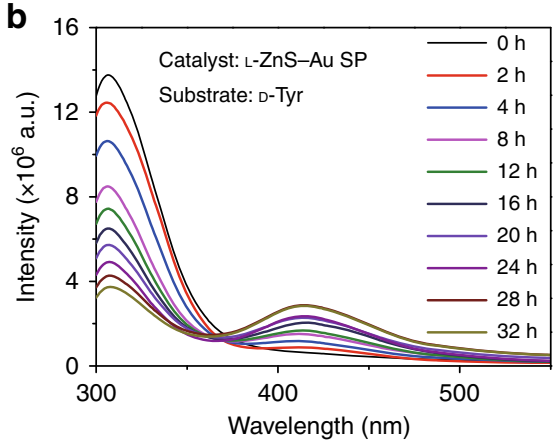

C
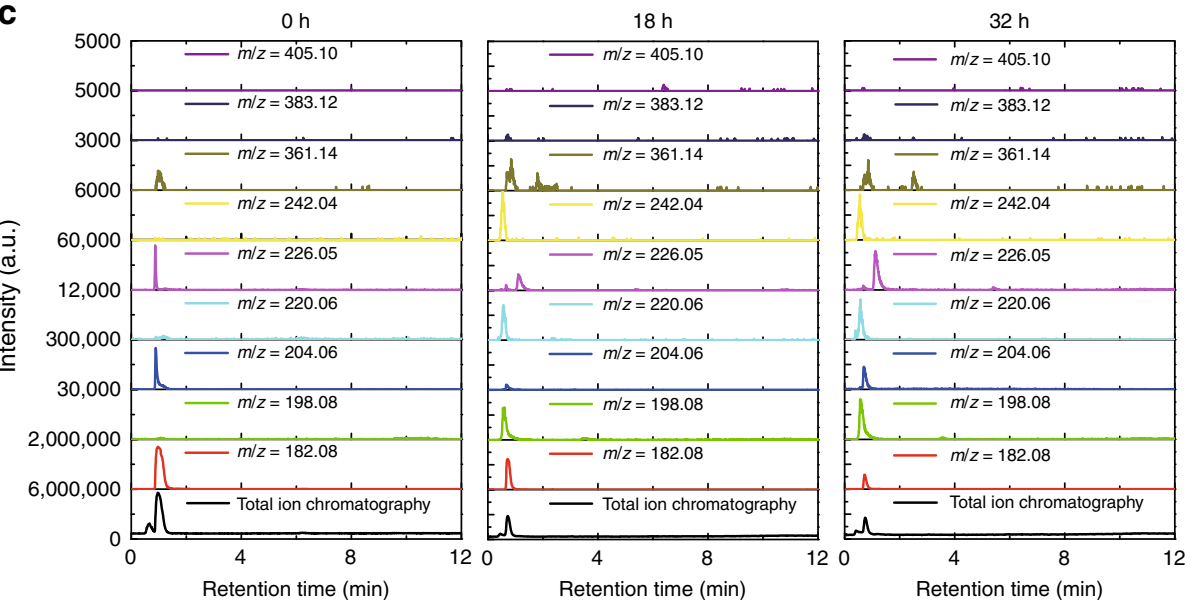

d
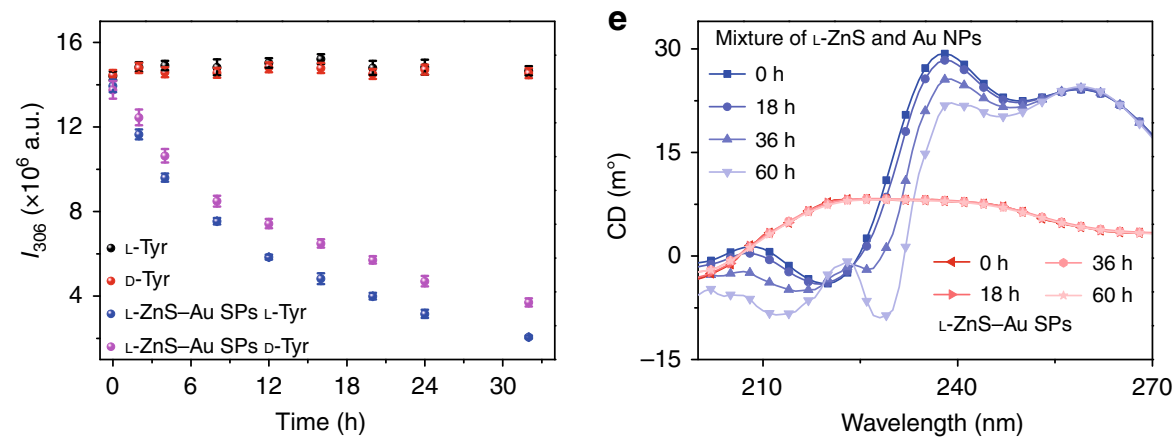

Fig. 5 Catalysis and stability of ZnS-Au SPs for photoinduced oxidative coupling of L-Tyr and D-Tyr. Fluorescence spectra of L-Tyr or D-Tyr after a L-Tyr with L-ZnS-Au SPs, b D-Tyr with L-ZnS-Au SPs being illuminated with different periods of time in the presence of ZnS-Au SPs. $\mathbf{c}$ Extracted ion chromatography (EIC) of L-Tyr and Tyr-related products of L-ZnS-Au SP L-Tyr samples obtained at 0, 18, and $32 \mathrm{~h}, \mathrm{~m} / \mathrm{z}=405.10,383.12$, and 361.14, and $\mathrm{m} / \mathrm{z}=242.04$, 220.06, and 198.08 are attributed to DOPA-Na $+\mathrm{Na}^{+}, \mathrm{DOPA}+\mathrm{Na}^{+}$, and DOPA $+\mathrm{H}^{+}$and diTyr-Na $+\mathrm{Na}+$, diTyr $+\mathrm{Na}^{+}$and diTyr $+\mathrm{H}^{+}$, respectively. $\mathbf{d}$ The dependence of PL intensity at $306 \mathrm{~nm}$ on photocatalytic reaction time with L-ZnS-Au SPs for different Tyr enantiomers. e CD spectra of the mixtures of ZnS and Au NPs, ZnS-Au SPs, after UV illumination (115 V, 50/60 Hz, $0.16 \mathrm{~A})$ for different times

difficult ${ }^{40,41}$ due to low chiral bias and significant due to variety of biomedical needs ${ }^{41}$. The problems with enantioselectivity are particularly noticeable for low molecular weight phenols as opposed to large molecular ${ }^{42}$ weight polycyclic aryls ${ }^{43}$. As such, optical purity of just a few percent is typically achieved even with complex metalorganic catalysts for diverse catalytic substrates ${ }^{42}$. In the majority of cases, however, no enantioselectivity was reported $^{40,41}$ and poor enantioselectivity of these reactions was pointed out even for sophisticated biomimetic catalysts ${ }^{42}$.

Incorporation of $\mathrm{Au}$ NPs to realize multicomponent SPs further enhanced photocatalytic conversion of the substrates. Besides improved electron-hole separation, more efficient light absorption by the assemblies via plasmonic effects and the formation of the NP junctions can facilitate both the electron transport, while retaining enantioselectivity. Concomitantly, photodecomposition under light is an essential problem of the chiral catalysts of any chemical type. Improving the resilience of chiral photocatalysts against photodegradation is an important advancement in the field that facilitates their practical implementation.

One can expect SPs to be a convenient platform for engineering catalysts using different NPs as functional modules. SPs provide a new approach to design a family of chiral catalysts conceptually different from those considered before in the field of homogeneous and heterogeneous chiral catalysts. Permutations of different NPs that can be combined in modular SPs is nearly endless. The development of more specific and sophisticated SPs that can replicate multiple other functions of biological nanoassemblies is anticipated. 
a

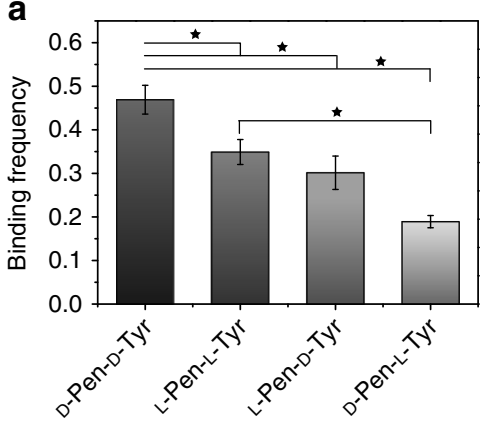

b

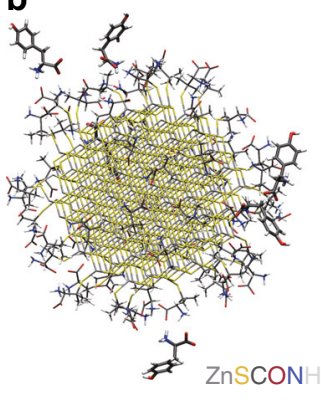

vigorous stirring, $1.6 \times 10^{-4} \mathrm{~mol}$ thioacetamide dissolved in water was added and then degassed for $30 \mathrm{~min}$ by argon bubbling. After that, the solution was heated for $30 \mathrm{~min}$ at $95^{\circ} \mathrm{C}, 6 \mathrm{~mL}$ of a concentrated dispersion of Au NPs was added and the volume brought to $100 \mathrm{~mL}$. The $\mathrm{pH}$ value of the concentrated Au NPs must be adjusted to 8.5 before mixing. The resulting mixture was heated to reflux at $95^{\circ} \mathrm{C}$ under flowing argon, and the final Au-ZnS SPs were obtained after $4 \mathrm{~h}$ of heating.

Photocatalysis with chiral ZnS SPs and ZnS-Au SPs. Solutions of L-Tyr and DTyr $\left(0.4 \mathrm{mg} \mathrm{mL}^{-1}\right)$ were prepared, and synthesized ZnS SPs and ZnS-Au SPs were washed three times with deionized water, and then dispersed in water. Solutions of L-ZnS SPs, D-ZnS SPs, and L-ZnS-Au SPs, assembled with the same concentration of ZnS NPs, were mixed with the same volume of $0.4 \mathrm{mg} \mathrm{mL}^{-1} \mathrm{~L}-\mathrm{Tyr}$ or D-Tyr. Control samples were prepared by mixing the same amount of water with L-Tyr or D-Tyr. Samples of L-Tyr, D-Tyr, L-ZnS SPs with L-Tyr, D-ZnS SPs with D-Tyr, L-ZnS SPs with D-Tyr, D-ZnS SPs with L-Tyr, L-ZnS-Au SPs with L-Tyr, and L-ZnS-Au SPs with D-Tyr were mixed thoroughly and the $\mathrm{pH}$ was adjusted to 4.7 or 8.0 before illumination. The prepared samples were illuminated with a mercury lamp for the desired time. Catalytic samples were continually stirred during illumination. SPs and solutions were separated by ultra-filtration, and filtered solutions were used for fluorescence and MS analyses. HPLC-MS measurements were performed using a $20-\mu \mathrm{L}$ injection volume, at testing $\mathrm{m} / \mathrm{z} 405.10,383.12,361.14,242.04,226.05$, $220.06,204.06,198.08$, and 182.08 that corresponded to diTyr- $\mathrm{Na}+\mathrm{Na}^{+}$, diTyr + $\mathrm{Na}^{+}, \operatorname{diTyr}+\mathrm{H}^{+}$, DOPA-Na $+\mathrm{Na}^{+}$, Tyr- $\mathrm{Na}+\mathrm{Na}^{+}, \mathrm{DOPA}+\mathrm{Na}^{+}, \mathrm{Tyr}+\mathrm{Na}^{+}$, $\mathrm{DOPA}+\mathrm{H}^{+}$, and $\mathrm{Tyr}+\mathrm{H}^{+}$ highlighted with green dotted lines and water molecules omitted for clarityANOVA analysis was used to explore significant differences of means comparison using the Fisher Test. The symbol * denotes the pairs with $P$ value $<0.05$ indicating significant difference

\section{Methods}

Reagents. $\mathrm{Zn}\left(\mathrm{ClO}_{4}\right)_{2} \cdot 6 \mathrm{H}_{2} \mathrm{O}$, thioacetamide, hydrogen tetrachloroaurate (III) trihydrate $\left(\mathrm{HAuCl}_{4} \cdot 3 \mathrm{H}_{2} \mathrm{O}\right)$, glutathione, and $D$ - and $L$ - Tyr were purchased from Sigma-Aldrich. All chemicals were used as received. Milli-Q-deionized water was used for all the experiments.

Characterization methods and instruments. Assemblies were characterized using a JEOL 3011 HRTEM instrument (JEOL USA, Inc.), and a JEOL2100F was employed for HAADF and BF imaging in STEM mode. Elemental analysis was performed using an EDAX accessory linked to the JEOL 2100F instrument (JEOL USA, Inc.), equipped with a UVL-56 lamp without a filter $(115 \mathrm{~V}, 50 / 60 \mathrm{~Hz}$, $0.16 \mathrm{~A})$.

UV-Vis absorbance spectra were acquired using an Agilent 89090A instrument (Agilent Technologies, Santa Clara, CA, USA). CD analysis was performed using a JASCO J-815 instrument. The $\zeta$-potential and size distribution were measured with a Nano ZS Zetasizer instrument (Malvern Instruments, Malvern, Worcestershire, UK). For $\zeta$-potential, each sample was equilibrated for $2 \mathrm{~min}$ before measurement; all experiments were performed in triplicate, each measurement included 50 cycles, and a 15-s pause was included between runs.

MS analysis was performed on an Agilent 6520 Accurate-Mass Quadrupole Time-of-Flight (Q-TOF) LC-MS instrument (Agilent Technologies, Santa Clara, CA, USA) operating in $\mathrm{ESI}^{+}$ion mode. The nebulization gas was set to $500 \mathrm{~L} \mathrm{~h}^{-1}$ at a temperature of $332{ }^{\circ} \mathrm{C}$, the cone gas was set to $5 \mathrm{~L} \mathrm{~min}^{-1}$, and the nebulizer gas pressure was $45 \mathrm{psi}$. The capillary voltage was set to $3500 \mathrm{~V}$. Internal reference ion masses $[\mathrm{M}+\mathrm{H}]^{+}$at $121.05 \mathrm{Da}$ and $922.01 \mathrm{Da}$ were enabled, and a detection window of $200 \mathrm{ppm}$ with a minimum height of 1000 counts was used. The results shown in Fig. 5c were obtained using an InfinityLab Poroshell 120 HILIC-Z HPLC column $(2.1 \times 50 \mathrm{~mm}, 2.7 \mu \mathrm{m}$ internal diameter $)$.

Synthesis of chiral ZnS SPs. For chiral SPs, $1.36 \times 10^{-3} \mathrm{~mol}$ of L-, D-, or $\mathrm{rac}$-Pen and $1.7 \times 10^{-4} \mathrm{~mol} \mathrm{Zn}\left(\mathrm{ClO}_{4}\right)_{2} \cdot 6 \mathrm{H}_{2} \mathrm{O}$ were dissolved in $86 \mathrm{~mL}$ of water, the $\mathrm{pH}$ was adjusted to 8.5 by adding $\mathrm{NaOH}$, and the solution was degassed by argon bubbling for $30 \mathrm{~min}$. A $1-\mathrm{mL}$ sample of $0.2 \mathrm{M}$ thioacetamide was quickly injected under vigorous stirring and the mixture was incubated at $95^{\circ} \mathrm{C}$ in a reflux column system. Samples were taken at different times for measurement and characterization. Penstabilized SPs were obtained after $3 \mathrm{~h}$ of heating.

Synthesis of chiral gold NPs. Briefly, $50 \mathrm{~mL}$ of $4.8 \mathrm{mM}$ glutathione (GSH) solution was mixed with $300 \mu \mathrm{L}$ of $0.5 \mathrm{M} \mathrm{HAuCl}_{4}$ under vigorous stirring. The mixture was heated at $95^{\circ} \mathrm{C}$ in an oil bath to obtain chiral gold NPs. The synthesized GSH-Au NPs were purified by placing in a centrifuge at $16,200 \times g$ to remove large aggregates after the reaction. The supernatant was further purified by adjusting the solution to $\mathrm{pH} 3.5$, adding 1 volume of ethanol (gold NPs solution: ethanol $=1: 1$ ), isolated using a centrifuge at $5000 \times g$ for $5 \mathrm{~min}$, and the supernatant was discarded. Precipitates were resuspended in $20 \mathrm{~mL}$ of water.

Synthesis of chiral ZnS-Au SPs. For chiral ZnS-Au SPs, $13.6 \times 10^{-4} \mathrm{~mol}$ of L-Pen and $1.7 \times 10^{-4} \mathrm{~mol}$ of $\mathrm{Zn}\left(\mathrm{ClO}_{4}\right)_{2} \cdot 6 \mathrm{H}_{2} \mathrm{O}$ were dissolved in water and the $\mathrm{pH}$ was adjusted to 8.5 . The solution was degassed by argon bubbling for $30 \mathrm{~min}$. Under

Enantioselectivity of chiral SPs for rac-Tyr. L-ZnS, D-ZnS, rac-ZnS, and L-ZnS$\mathrm{Au}$ SPs were incubated with $0.4 \mathrm{mg} \mathrm{mL}^{-1} \mathrm{rac}$-Tyr for $6 \mathrm{~h}$ with stirring. Sodium chloride was then added to obtain a concentration of $0.5 \mathrm{M}$ in order to cause the sedimentation of SPs. As the SPs settled to the bottom, no centrifugation was needed. The supernatant from the top part of the vial was diluted with ultrapure water to avoid saturation of the detectors. The diluted samples of the supernatant were used for absorbance and $\mathrm{CD}$ measurements.

Construction of molecular models and simulation setup. All-atom models of the ZnS NP, (L)-Pen, (D)-Pen, (L)-Tyr, (D)-Tyr, and water were built using Materials Studio. A NP model of $3 \mathrm{~nm}$ size $\left.\left(\mathrm{Zn}_{369} \mathrm{~S}_{348} \mathrm{~S} \text { (Ligand) }\right)_{54}\right)$ was created from a multiple of the unit cell of sphalerite (ZnS) and application of a spherical cutoff. Atomic charges were assigned with charge increments of $\pm 0.25 \mathrm{e}$ per bonded neighbor, corresponding to an atomic charge of $+1.0 \mathrm{e}$ for $\mathrm{Zn}$ and $-1.0 \mathrm{e}$ for $\mathrm{S}$ in bulk ZnS, and accordingly reduced charges on the surface. Fifty-four (L or D)-Pen ligands were bound via thiol linkages to the surface and randomly spread (packing density $\left.\sim 1.9 \mathrm{~nm}^{-2}\right)^{62}$. The protonation states of carboxylic acid groups and amine groups in (L or D)-Pen and ( $\mathrm{L}$ or D)-Tyr were adjusted to $\mathrm{COO}^{-}$and $\mathrm{NH}_{3}{ }^{+}$to represent neutral conditions of $\mathrm{pH} \simeq 7$ as in experiment.

Simulations were performed for four systems, including (1) L-Pen-ZnS-L-Tyr, (2) L-Pen-ZnS-D-Tyr, (3) D-Pen-ZnS-L-Tyr, and (4) D-Pen-ZnS-D-Tyr. The dimensions for each box were the same, about $70 \times 70 \times 70 \AA^{3}$, and all simulation systems were overall charge-neutral. The complete start structures consisted of one ZnS NP modified with (D) or (L) Pen, 8 (D) or (L) Tyr molecules in solution, and 10229 water molecules. The Tyr molecules were evenly placed in the box with $\sim 10$ $\AA$ distance from the Pen-ZnS NP.

Force field. We employed the CHARMM-Interface force field (CHARMM-IFF) including new parameters for ZnS (Table 1$)^{63}$ and existing CHARMM36 ${ }^{64}$ parameters for (L or D)-Pen and ( $\mathrm{L}$ or D)-Tyr. The new force field parameters for ZnS describe the expected amount of covalent and ionic bonding ${ }^{64,65}$ and reproduce the lattice parameters $\left(5.4093 \AA\right.$ ) and density $\left(4.09 \mathrm{~g} \mathrm{~cm}^{-3}\right)$ from XRD data (Supplementary Fig. 11) with $0.2 \%$ deviation $(5.394 \AA)$ and $0.7 \%$ deviation $\left(4.12 \mathrm{~g} \mathrm{~cm}^{-3}\right)$,

\section{Table 1 Forcefield parameters for ZnS (sphalerite)}

\begin{tabular}{|llll|} 
I. Nonbond & Charge (e) & $\boldsymbol{\sigma}(\mathbf{p m})$ & $\boldsymbol{\varepsilon}\left(\mathbf{k c a l ~} \mathbf{~ m o l}^{-\mathbf{1}}\right)$ \\
\hline Zn & +1.00 & 185 & 0.05 \\
$\mathrm{~S}$ & -1.00 & 448 & 0.5 \\
II. Bond & $r_{0}(\mathrm{pm})$ & $k_{\mathrm{r}}\left(\mathrm{kcal} \mathrm{mol}^{-1} \AA^{-2}\right)$ \\
Zn-S & 2.41 & 525 \\
Zn-S (ligand) & 2.41 & 525 \\
III. Angles & $\theta_{0}\left(^{\circ}\right)$ & $k_{\theta}\left(\mathrm{kcal} \mathrm{mol}^{-1} \mathrm{rad}^{-2}\right)$ \\
Zn-S-Zn & 109.5 & 200 \\
S-Zn-S & 109.5 & 200 \\
S-Zn-S (ligand) & 109.5 & 200 \\
Zn-S-C (ligand) & 100.0 & 0 \\
\hline Note: No dihedral potentials were necessary for bonds inside the ZnS NP (zero)
\end{tabular}


respectively, at $298.15 \mathrm{~K}$ and $1 \mathrm{~atm}$. The Lennard-Jones parameters approximated surface and interfacial energies of $\mathrm{ZnS}$ (not tested in detail) ${ }^{66}$.

Simulation protocol. Each system was initially subjected to 1000 time steps energy minimization to remove atomic close contacts. The configurations were then subjected to an initial 2-ns equilibration period of molecular dynamics simulation in the NPT ensemble at 298.15 K and 1.013 MPa using the Nanoscale Molecular Dynamics program (NAMD) ${ }^{67}$, followed by longer simulation times of $50 \mathrm{~ns}$ to thoroughly sample conformations and thermodynamic averages. All atoms were flexible during the simulations, the time step was $1 \mathrm{fs}$, temperature controlled by the Langevin thermostat, and a damping coefficient of $1 \mathrm{ps}^{-1}$. Van-der-Waals interactions were treated with a spherical cutoff of $12 \AA$ in the summation of pairwise Lennard-Jones interactions, and the summation of Coulomb interactions was carried out using the Particle Mesh Ewald (PME) method with a high accuracy of $10^{-6} \mathrm{kcal} \mathrm{mol}^{-1}$ throughout the equilibration and production runs.

Analysis and calculation of binding frequency. The conformations and dynamics were visually analyzed using the Visual Molecular Dynamics (VMD) program ${ }^{68}$. Binding was defined as uninterrupted proximity of Tyr molecules to the Pen-ZnS NP within $5 \AA$ for 100 ps or more. The binding frequency was calculated from the number count of binding frames, that is, the sum of the number $n_{i}$ of Tyr molecules bound in each frame $i$ over all frames $N$, relative to the total number of frames $N$ and 8 Tyr molecules:

$$
\text { Binding frequency }=\frac{\sum_{i=1}^{N} n_{i}}{8 \times N} .
$$

Uncertainties are given using block averages of the binding frequency over major portions of the total simulation time.

\section{Data availability}

The data that support the findings of this study are available from the corresponding author upon reasonable request.

Received: 17 January 2019 Accepted: 22 August 2019

Published online: 23 October 2019

\section{References}

1. Palade, G. Intracellular aspects of the process of protein synthesis. Science 189, 867-867 (1975).

2. Eschenmoser, A. \& Loewenthal, E. Chemistry of potentially prebiological natural products. Chem. Soc. Rev. 21, 1 (1992).

3. Lidmar, J., Mirny, L. \& Nelson, D. R. Virus shapes and buckling transitions in spherical shells. Phys. Rev. E 68, 051910 (2003).

4. Scott, C. C., Vacca, F. \& Gruenberg, J. Endosome maturation, transport and functions. Semin. Cell Dev. Biol. 31, 2-10 (2014).

5. Tanaka, S. et al. Atomic-level models of the bacterial carboxysome shell. Science 319, 1083-1086 (2008).

6. Brown, W. J., Shannon, W. A. \& Snell, W. J. Specific and azurophilic granules from rabbit polymorphonuclear leukocytes. I. Isolation and characterization of membrane and content subfractions. J. Cell Biol. 96, 1030-1039 (1983).

7. Chandler, D. E., Strümpfer, J., Sener, M., Scheuring, S. \& Schulten, K. Light harvesting by lamellar chromatophores in Rhodospirillum photometricum. Biophys. J. 106, 2503-2510 (2014).

8. Settembre, C., Fraldi, A., Medina, D. L. \& Ballabio, A. Signals from the lysosome: a control centre for cellular clearance and energy metabolism. Nat. Rev. Mol. Cell Biol. 14, 283-296 (2013).

9. Buchan, J. R., Kolaitis, R.-M., Taylor, J. P. \& Parker, R. Eukaryotic stress granules are cleared by autophagy and Cdc48/VCP function. Cell 153, 1461-1474 (2013).

10. van der Pol, E., Boing, A. N., Harrison, P., Sturk, A. \& Nieuwland, R. Classification, functions, and clinical relevance of extracellular vesicles. Pharmacol. Rev. 64, 676-705 (2012).

11. Yeates, T. O., Kerfeld, C. A., Heinhorst, S., Cannon, G. C. \& Shively, J. M. Protein-based organelles in bacteria: carboxysomes and related microcompartments. Nat. Rev. Microbiol. 6, 681-691 (2008).

12. Shen, X., Zhang, K. \& Kaufman, R. J. The unfolded protein response - a stress signaling pathway of the endoplasmic reticulum. J. Chem. Neuroanat. $\mathbf{2 8}$ 79-92 (2004).

13. Harding, H. P., Zhang, Y. \& Ron, D. Protein translation and folding arecoupledbyan endoplasmic-reticulum- resident kinase. Nature 397, 271-274 (1999).

14. Webber, M. J. \& Langer, R. Drug delivery by supramolecular design. Chem Soc. Rev. 46, 6600-6620 (2017).
15. Wu, M. X. \& Yang, Y. W. Metal-organic framework (MOF)-based drug/cargo delivery and cancer therapy. Adv. Mater. 29, 1-20 (2017).

16. Han, D. et al. DNA origami with complex curvatures in three-dimensional space. Science 332, 342-346 (2011).

17. Zhao, Z. et al. Nanocaged enzymes with enhanced catalytic activity and increased stability against protease digestion. Nat. Commun. 7, 10619 (2016).

18. Sørensen, M. A., Fehler, A. O. \& Lo Svenningsen, S. Transfer RNA instability as a stress response in Escherichia coli: rapid dynamics of the tRNA pool as a function of demand. RNA Biol. 15, 586-593 (2018).

19. Lindahl, T. Instability and decay of the primary structure of DNA. Nature 362, 709-715 (1993).

20. Kotov, N. A. Inorganic nanoparticles as protein mimics. Science 330, 188-189 (2010).

21. Xia, Y. et al. Supraparticles from polydisperse nanoparticles. Nat. Nanotechnol. 6, 580-587 (2011)

22. Park, W. M. \& Champion, J. A. Colloidal assembly of hierarchically structured porous supraparticles from flower-shaped protein-inorganic hybrid nanoparticles. ACS Nano 10, 8271-8280 (2016).

23. Grason, G. M. Perspective: geometrically frustrated assemblies. J. Chem. Phys 145, 110901 (2016)

24. Xia, Y. et al. Self-assembly of self-limiting monodisperse supraparticles from polydisperse nanoparticles. Nat. Nanotechnol. 6, 580-587 (2011).

25. Yang, M. et al. Self-assembly of nanoparticles into biomimetic capsid-like nanoshells. Nat. Chem. 9, 287-294 (2017).

26. Feng, W. et al. Assembly of mesoscale helices with near-unity enantiomeric excess and light-matter interactions for chiral semiconductors. Sci. Adv. 3, e1601159 (2017).

27. Bryant, D. A. \& Frigaard, N.-U. Prokaryotic photosynthesis and phototrophy illuminated. Trends Microbiol. 14, 488-496 (2006).

28. Kober, L., Zehe, C. \& Bode, J. Development of a novel ER stress based selection system for the isolation of highly productive clones. Biotechnol. Bioeng. 109, 2599-2611 (2012).

29. Ma, W. et al. Chiral inorganic nanostructures. Chem. Rev. 117, 8041-8093 (2017)

30. Noyori, R. \& Takaya, H. BINAP: an efficient chiral element for asymmetric catalysis. Acc. Chem. Res. 23, 345-350 (1990).

31. Hu, A., Yee, G. T. \& Lin, W. Magnetically recoverable chiral catalysts immobilized on magnetite nanoparticles for asymmetric hydrogenation of aromatic ketones. J. Am. Chem. Soc. 127, 12486-12487 (2005).

32. Oila, M. J. Chirally modified gold nanoparticles: nanostructured chiral ligands for catalysis. Arkivoc 2006, 76-83 (2006).

33. Gross, E. et al. Asymmetric catalysis at the mesoscale: gold nanoclusters embedded in chiral self-assembled monolayer as heterogeneous catalyst for asymmetric reactions. J. Am. Chem. Soc. 135, 3881-3886 (2013).

34. Sawai, K., Tatumi, R., Nakahodo, T. \& Fujihara, H. Asymmetric SuzukiMiyaura coupling reactions catalyzed by chiral palladium nanoparticles at room temperature. Angew. Chem. Int. Ed. 47, 6917-6919 (2008).

35. Hou, J. et al. Catalyzed and electrocatalyzed oxidation of 1 -tyrosine and 1 -phenylalanine to dopachrome by nanozymes. Nano Lett. 18, 4015-4022 (2018).

36. Wang, Y. et al. Atomically precise alkynyl-protected metal nanoclusters as a model catalyst: observation of promoting effect of surface ligands on catalysis by metal nanoparticles. J. Am. Chem. Soc. 138, 3278-3281 (2016).

37. Tao, Y., Ju, E., Ren, J. \& Qu, X. Bifunctionalized mesoporous silica-supported gold nanoparticles: intrinsic oxidase and peroxidase catalytic activities for antibacterial applications. Adv. Mater. 27, 1097-1104 (2015).

38. Chen, J. et al. One-pot synthesis of CdS nanocrystals hybridized with singlelayer transition-metal dichalcogenide nanosheets for efficient photocatalytic hydrogen evolution. Angew. Chem. Int. Ed. 54, 1210-1214 (2015).

39. Hingorani, K. et al. Photo-oxidation of tyrosine in a bio-engineered bacterioferritin 'reaction centre'-a protein model for artificial photosynthesis. Biochim. Biophys. Acta Bioenerg. 1837, 1821-1834 (2014).

40. Eickhoff, H., Jung, G. \& Rieker, A. Oxidative phenol coupling - tyrosine dimers and libraries containing tyrosyl peptide dimers. Tetrahedron 57, 353-364 (2001)

41. Reid, L. O. et al. A novel synthetic approach to tyrosine dimers based on pterin photosensitization. Dyes Pigm. 147, 67-74 (2017)

42. Feringa, B. \& Wynberg, H. Biomimetic asymmetric oxidative coupling of phenols. Bioorg. Chem. 7, 397-408 (1978).

43. Zoia, L., Bruschi, M., Orlandi, M., Tolppa, E.-L. \& Rindone, B. Asymmetric biomimetic oxidations of phenols: the mechanism of the diastereo- and enantioselective synthesis of thomasidioic acid. Molecules 13, 129-148 (2008).

44. Wattanakit, C. et al. Enantioselective recognition at mesoporous chiral metal surfaces. Nat. Commun. 5, 3325 (2014).

45. Banerjee-Ghosh, K. et al. Separation of enantiomers by their enantiospecific interaction with achiral magnetic substrates. Science 360, 1331-1334 (2018).

46. Article, R. A review on zinc sulphide nanoparticles: from synthesis, properties to applications. J. Bioelectron. Nanotechnol. 1, 1-5 (2016). 
47. Zhou, Y. et al. Biomimetic hierarchical assembly of helical suprapartcles from chiral nanoparticles. ACS Nano 10, 3248-3256 (2016).

48. MacLean, M. W. A. et al. Unraveling inter- and intrachain electronics in polythiophene assemblies mediated by coordination nanospaces. Angew. Chem. Int. Ed. 55, 708-713 (2016).

49. Yu, D. et al. Scalable synthesis of hierarchically structured carbon nanotube-graphene fibres for capacitive energy storage. Nat. Nanotechnol. 9, 555-562 (2014).

50. Garcia-Viloca, M. How enzymes work: analysis by modern rate theory and computer simulations. Science 303, 186-195 (2004).

51. Mahmoud, S. F. \& Bialkowski, S. E. Laser-excited fluorescence of dityrosine. Appl. Spectrosc. 49, 1669-1676 (1995).

52. Heineckes, J. W., Li, W., Daehnke, H. L. \& Goldstein, J. A. Dityrosine, a specific marker of oxidation, is synthesized by the myeloperoxidase-hydrogen peroxide system of human neutrophils and macrophagesdityrosine. J. Biol. Chem. 268, 4069-4077 (1993).

53. Wood, A. T. \& Hall, M. R. Reversed-phase high-performance liquid chromatography of catecholamines and indoleamines using a simple gradient solvent system and native fluorescence detection. J. Chromatogr. B Biomed. Sci. Appl. 744, 221-225 (2000).

54. Lehrer, S. S. \& Fasman, G. D. Ultraviolet irradiation effects in poly-L-tyrosine and model compounds. Identification of bityrosine as a photoproduct. Biochemistry 6, 757-767 (1967).

55. Cole, S. H. et al. Two functional but noncomplementing Drosophila tyrosine decarboxylase genes: distinct roles for neural tyramine and octopamine in female fertility. J. Biol. Chem. 280, 14948-14955 (2005).

56. Yutthalekha, T., Warakulwit, C., Limtrakul, J. \& Kuhn, A. Enantioselective recognition of DOPA by mesoporous platinum imprinted with mandelic acid. Electroanalysis 27, 2209-2213 (2015).

57. Zhang, Y. et al. One-step synthesis of chiral carbon quantum dots and their enantioselective recognition. RSC Adv. 6, 59956-59960 (2016).

58. Wang, F., Gong, W., Wang, L. \& Chen, Z. Selective recognition of dtryptophan from $\mathrm{d} / \mathrm{l}$-tryptophan mixtures in the presence of $\mathrm{Cu}(\mathrm{II})$ by electropolymerized l-lysine film. Anal. Biochem. 492, 30-33 (2016).

59. Harding, J. R., Amanchukwu, C. V., Hammond, P. T. \& Shao Horn, Y. Instability of poly(ethylene oxide) upon oxidation in lithium air batteries. $J$. Phys. Chem. C 119, 6947-6955 (2015).

60. Al-Janabi, N., Alfutimie, A., Siperstein, F. R. \& Fan, X. Underlying mechanism of the hydrothermal instability of $\mathrm{Cu} 3(\mathrm{BTC}) 2$ metal-organic framework. Front. Chem. Sci. Eng. 10, 103-107 (2016).

61. Zhang, Q. et al. Supernatant organics from anaerobic digestion after thermal hydrolysis cause direct and/or diffusional activity loss for nitritation and anammox. Water Res. 143, 270-281 (2018).

62. Heinz, H. et al. Nanoparticle decoration with surfactants: molecular interactions, assembly, and applications. Surf. Sci. Rep. 72, 1-58 (2017)

63. Heinz, H., Lin, T.-J., Kishore Mishra, R. \& Emami, F. S. Thermodynamically consistent force fields for the assembly of inorganic, organic, and biological nanostructures: the INTERFACE force field. Langmuir 29, 1754-1765 (2013).

64. Huang, J. \& MacKerell, A. D. CHARMM36 all-atom additive protein force field: validation based on comparison to NMR data. J. Comput. Chem. 34, 2135-2145 (2013).

65. Liu, H. et al. Interaction of substituted poly(phenyleneethynylene)s with ligand-stabilized CdS nanoparticles. J. Mater. Chem. A 2, 8705-8711 (2014).

66. Chen, J. et al. Building two-dimensional materials one row at a time: avoiding the nucleation barrier. Science 362, 1135-1139 (2018).

67. Phillips, J. C. et al. Scalable molecular dynamics with NAMD. J. Comput. Chem. 26, 1781-1802 (2005).

68. Humphrey, W., Dalke, A. \& Schulten, K. VMD: visual molecular dynamics. J. Mol. Graph. 14, 33-38 (1996).

\section{Acknowledgements}

The central part of this work was supported by the NSF project "Energy- and CostEfficient Manufacturing Employing Nanoparticles” NSF 1463474. NSF 1566460 Nanospiked Particles for Photocatalysis. This work was also supported by the National Natural Science Foundation of China (21631005, 21673104, 21522102, 21503095). The authors also thank NSF 1538180 Layered Composites from Branched Nanofibers for Lithium Ion Batteries for partial support to the project; MURI: Department of Army W911NF-10-1-0518 Reconfigurable Matter from Programmable Colloids; AFOSR FA9550-16-1-0265, UM 088163- Nanocomposite Ion Conductors for Thin Film Batteries; $\mathrm{MC}^{2}$ for its assistance with electron microscopy, and for the NSF grant \#DMR-9871177 for funding of the JEOL 2010F analytical electron microscope used in this work. H.H. acknowledges grant DMREF 1623947, "DMREF/Collaborative Research: Design and Testing of Nanoalloy Catalysts in 3D Atomic Resolution". This work utilized the Summit supercomputer, a joint effort of the University of Colorado Boulder and Colorado State University, which is supported by the National Science Foundation (ACI-1532235 and ACI-1532236), and the Argonne Leadership Computing Facility, which is a DOE Office of Science User Facility supported under Contract DEAC02-06CH11357.

\section{Author contributions}

S.L. performed the experiments and processed the experimental data under the supervision of C.X., L.X., and N.A.K. and drafted the manuscript. N.R., J.L. and H.H. carried out MD calculations. C.X., L.X. and N.A.K. participated in the whole process, including the development of the principles and experimental design, data analysis, and manuscript preparation. N. A. K. initiated the idea and designed the study. All authors participated in discussions and writing the manuscript.

\section{Additional information}

Supplementary Information accompanies this paper at https://doi.org/10.1038/s41467019-12134-4.

Competing interests: The authors declare no competing interests.

Reprints and permission information is available online at http://npg.nature.com/ reprintsandpermissions/

Peer review information Nature Communications thanks Dario Pasini, and other, anonymous, reviewer(s) for their contribution to the peer review of this work.

Publisher's note Springer Nature remains neutral with regard to jurisdictional claims in published maps and institutional affiliations.

Open Access This article is licensed under a Creative Commons Attribution 4.0 International License, which permits use, sharing, adaptation, distribution and reproduction in any medium or format, as long as you give appropriate credit to the original author(s) and the source, provide a link to the Creative Commons license, and indicate if changes were made. The images or other third party material in this article are included in the article's Creative Commons license, unless indicated otherwise in a credit line to the material. If material is not included in the article's Creative Commons license and your intended use is not permitted by statutory regulation or exceeds the permitted use, you will need to obtain permission directly from the copyright holder. To view a copy of this license, visit http://creativecommons.org/ licenses/by/4.0/

(C) The Author(s) 2019 